\title{
ACTITUDES DE LOS ESTUDIANTES EN ESCUELAS SEGREGADAS Y EN ESCUELAS INCLUSIVAS, HACIA LA TOLERANCIA SOCIAL Y LA CONVIVENCIA ENTRE PARES
}

\section{Iván Ortiz ${ }^{1}$}

\begin{abstract}
RESUMEN
A partir de la publicación de la Ley No 20.845, acerca de inclusión social en educación, se espera que aumente la mixtura social al interior de las escuelas, hoy muy segregadas. La teoría del contacto intergrupal, en psicología social, sugiere que ocurren efectos positivos -disminución del prejuicio y de las actitudes hostiles- a partir del contacto directo entre los grupos sociales en tensión. Este artículo aporta información de lo que ocurre en escuelas segregadas y en escuelas inclusivas respecto de ciertas actitudes como la tolerancia social, la confianza en la gente y la convivencia entre pares. Se usaron los datos chilenos del Estudio Internacional de Educación Cívica y Ciudadanía realizado en 2009. En general, los resultados mostraron que los estudiantes de escuelas inclusivas tienen disposiciones actitudinales distintas de las de sus pares de segregadas aventajadas, pero no tan distintas de las de sus pares de segregadas pobres.
\end{abstract}

Palabras clave: actitudes sociales, educación cívica, relaciones intergrupales, segregación escolar

\section{STUDENT ATTITUDES TOWARDS SOCIAL TOLERANCE AND COEXISTENCE AMONG PEERS, IN SEGREGATED SCHOOLS AND INCLUSIVE SCHOOLS}

\begin{abstract}
It is expected that the recent legislation, No. 20.845, on social inclusion in education, will increase the social mix within schools, which are currently highly segregated. The intergroup contact theory in social psychology suggests that positive effects occur, such as a decrease in prejudice and hostile attitudes, when social groups in conflict have direct contact. . This article provides some evidence of what happens in segregated schools and inclusive schools with respect to certain attitudes such as social tolerance, trust in people and coexistence among peers. Chilean data from the International Civic and Citizenship Study carried out in 2009, were used. In general, the results showed that students in inclusive schools have different attitudes from those of privileged segregated schools, but not so different from those who attend disadvantaged segregated schools.
\end{abstract}

Keywords: civic education, intergroup relations, school segregation, social attitudes.

1 Facultad de Educación, Universidad Alberto Hurtado, Santiago, Chile. Contacto: ivanortizc@gmail.com 


\section{Introducción}

Es sabido que la escuela no tiene que ver solo con la transmisión de conocimiento disciplinario. El respeto hacia la diversidad social, la tolerancia, la identidad común y otros valores ciudadanos siempre han sido objetivos importantes que el Estado y la sociedad promueven a través de la institución escolar (Iaes \& Delich, 2009). Todo esto en pos de la cohesión social entre los ciudadanos, factor necesario para la vida en democracia. Consecuentemente, se aprecia un creciente interés académico y político por conocer el desempeño de la escuela en estas dimensiones de formación. La Ley No 20.529, que trata del aseguramiento de la calidad de la educación, estableció indicadores de calidad que deben ser medidos y monitoreados en las escuelas, entre los cuales figuran la participación y formación ciudadana y el clima de convivencia escolar, además de estándares de aprendizaje de las asignaturas tradicionales ${ }^{2}$.

Por su parte, la International Association for the Evaluation of Educational Achievement (IEA) ha coordinado tres estudios internacionales en torno a la cultura cívica en los estudiantes ${ }^{3}$ : el de 1999, para estudiantes de 13 años (CIVID 1999²); el de 2000 para estudiantes de 17 años (CIVID 2000); y el de 2009, nuevamente para estudiantes de 13 años (ICCS 2009). Existe un cuarto estudio en desarrollo (ICCS 2016), que se aplicó en Chile y en otros países del hemisferio sur durante el segundo semestre del año 2015 (en los países del hemisferio norte se aplica durante 2016) y cuyos resultados estarán disponibles en el transcurso del año 2017. El informe internacional de ICCS 2009 describe los resultados globales de los 38 países participantes (Schultz, Ainley, Fraillon, Kerr \& Losito, 2010); existe otro informe más específico que se centra en los resultados de los seis países latinoamericanos participantes (Schulz, Ainley, Friedman \& Lietz, 2011). Aparte de los resultados en las pruebas cognitivas, los informes mencionados entregan información de una gran variedad de actitudes

2 Revisar dichos indicadores en el link agenciaeducacion.cl/coordinacion-sac/otrosindicadores-de-calidad-educatival

3 La IEA también desarrolla otros importantes estudios internacionales, tales como TIMSS, que evalúa Ciencias y Matemática.

4 Civic Education Study.

5 International Civic and Citizenship Study. 
de los estudiantes en torno a temas de ciudadanía y política. A pesar de la abundancia de resultados generales, los informes no profundizan en busca de relaciones más implícitas entre ellos. Tarea que queda en manos de investigadores sociales interesados en cómo la educación puede afectar positivamente el comportamiento cívico en sus países.

Los estudios internacionales de educación cívica han revelado que el aprendizaje del conocimiento cívico que se imparte en las escuelas está claramente relacionado con los antecedentes socioeconómicos de los estudiantes, en el mismo sentido en que lo está el rendimiento en otras materias, como lectura, matemáticas y ciencias. Algunas actitudes cívicas se ven, además, influenciadas por el origen social y disposiciones conductuales. Castillo y otros autores (2014a, 2014b) dejan ver su preocupación por la inequidad en la participación política formal asociada a la inequidad socioeconómica -participan más los sectores sociales favorecidos- una situación que Chile necesita superar, de modo que los sectores sociales desfavorecidos puedan trasladar demandas redistributivas a la arena política formal, mejorando así la calidad de la democracia. Se esperaría que la educación cívica en las escuelas contribuyese a esa superación, por la vía del conocimiento cívico impartido y por la vía de las prácticas de ciudadanía que allí se modelan y se promuevan.

Con este fin, Castillo y otros autores (2014a) analizaron resultados de la muestra chilena en ICCS 2009, reportando la influencia de diversas variables sobre la disposición de los estudiantes a la participación electoral cuando sean adultos, en un intento de averiguar si el reconocido peso del nivel socioeconómico sobre la participación electoral podía ser morigerado por otras influencias más cercanas a la experiencia escolar. Encontraron que el clima abierto a la discusión de asuntos políticos en la sala de clases -una de las variables afectivo-conductuales del estudio- podía jugar ese rol mediador. En otro artículo, los mismos autores (Castillo et al., 2014b) averiguaron si el peso del factor socioeconómico sobre la disposición a la participación electoral futura disminuyó o no entre 1999 y 2009, dado los cambios sociales y curriculares acaecidos en el país en ese lapso de tiempo; no obstante, sus resultados confirmaron la continuidad del peso de este factor entre las dos mediciones. 
Pero al mismo tiempo, en los estudios internacionales se ha encontrado poca relación entre el nivel socioeconómico (NSE) y varias actitudes y conductas ciudadanas de los estudiantes. Entre otras cosas, se halló que algunas de estas actitudes y disposiciones eran influenciadas por el hogar, de manera independiente del NSE (Amadeo, Torney-Purta, Lehmann, Husfeldt $\&$ Nikolova, 2002; Oswald \& Schulz, 2001; Schultz et al., 2010; Torney-Purta, Lehmann). Estos hallazgos sugieren que también la escuela puede ser un lugar propicio para la formación cívica de actitudes y conductas cívicas, por lo menos de aquellas que son menos influenciadas por el NSE de los estudiantes, lo cual abre oportunidades de compensación de la típica brecha de aprendizajes disciplinares entre estudiantes más y menos aventajados socialmente.

Una forma alternativa de explorar la relación entre la escuela, el NSE y las actitudes cívicas consiste en preguntarse por el efecto de la segregación social y su opuesto, la mixtura social de la escuela. ¿Es relevante para el desarrollo de ciertas actitudes cívicas el hecho de que las escuelas concentren estudiantes de un mismo NSE o, por el contrario, mezclen estudiantes de estatus diversos? Es una pregunta que cobra mayor relevancia a la luz de la Ley No 20.845, que estableció una serie de mecanismos para evitar la segregación escolar y promover la inclusión. Siguiendo el espíritu de esta ley, se espera que aumente la mixtura social al interior de las escuelas, que estudiantes de diferentes estratos socioeconómicos aprendan a conocerse mejor, a tolerarse y a deshacer los prejuicios negativos mutuos. El presente estudio indaga en qué medida la segregación social de la escuela, mayor o menor, está asociada con ciertas actitudes y conductas relacionadas con la (in)tolerancia social y la convivencia con los pares, actitudes cuya selección para este estudio se aclara en las páginas siguientes.

\section{Antecedentes teóricos y empíricos}

La segregación social de los sistemas educativos no tiene apoyo académico ni político, en la medida en que atenta contra uno de los bienes educacionales más promovidos en las últimas décadas: la equidad social y educativa. La Organisation for Economic Co- 
operation and Development (OECD), a través del programa PISA ${ }^{6}$, ha informado profusamente acerca de la extensión de la segregación en los países, su relación con el rendimiento académico y las medidas de política que la superarían. Los países latinoamericanos participantes en los estudios del rendimiento académico que lleva a cabo esta organización, presentan índices altos de segregación de sus sistemas escolares. De 63 países participantes en el estudio PISA 2012, las tres últimas posiciones en el índice de inclusión social ${ }^{7}$ pertenecen a Chile, Perú y México; un poco más arriba está Uruguay y Costa Rica, luego Brasil y Colombia. El país latinoamericano mejor posicionado es Argentina en el puesto 49 de 63, de mayor a menor inclusión social (OECD, 2013).

La segregación escolar tiene efectos negativos para los resultados académicos de los estudiantes concentrados en escuelas de bajo NSE, y positivos para aquellos reunidos en escuelas de alto NSE, hecho ampliamente descrito por la literatura y confirmado también para el caso de Chile (OECD, 2010; OECD, 2013, Valenzuela, Bellei y De los Ríos, 2009, entre otros). La investigación internacional ha podido comprobar, además, que la mixtura social en las escuelas tiene efectos positivos para el aprendizaje de los estudiantes de NSE vulnerable ${ }^{8}$, tema que en Chile ha sido poco investigado.

Pero las consecuencias de la segregación escolar no se limitarían al rendimiento desigual de los estudiantes favorecidos y desfavorecidos. Es razonable suponer que la segregación socioeconómica altera la calidad de la experiencia formativa de los alumnos en un sentido más amplio, al impedir que los estudiantes aprendan precozmente a convivir con respeto y tolerancia hacia los compañeros de clases sociales diferentes, y al impedir que la convivencia mutua genere principios de identificación social común, tan necesarios para el desarrollo democrático y la cohesión social (Peña, 2007).

6 Programme for International Student Assessment.

7 Este índice elaborado por PISA se refiere al porcentaje de la varianza total del estatus socioeconómico de los estudiantes de un país, encontrado al interior de las escuelas. Mientras más alto este porcentaje, más inclusiva es la escuela, porque en su interior conviven estudiantes de una amplia diversidad de estatus socioeconómico. En Finlandia y Noruega este índice supera el 90\%; en Chile alcanza el 47\%.

8 Ver Harris 2010, para una revisión relativamente reciente. 
La escuela segregada o la escuela inclusiva serían, entonces, dos espacios formativos potencialmente diferentes, por las dinámicas de interacción social que ocurrirían en su interior. Así, se presume que una escuela inclusiva sería un espacio formativo más apropiado para el aprendizaje de la tolerancia social, por cuanto pone en contacto directo a estudiantes de diverso origen social, generando oportunidades de encuentro y conocimiento mutuo, condición básica para tales aprendizajes. Pero por otra parte, hay que decir que los espacios donde conviven personas de pertenencias sociales diversas pueden ser también espacios de polarización social. El contacto directo entre estudiantes de grupos sociales diferentes puede dar origen al nacimiento o a la actualización de estereotipos y prejuicios mutuos, si estos se perciben como miembros de grupos distintos, hecho ampliamente demostrado en psicología social (Tajfel, 1984).

En 1954, Gordon Allport (citado por Hodson \& Hewstone, 2013) formuló la teoría del contacto intergrupal. Allí el autor sugirió que ocurren efectos positivos para las relaciones intergrupales -disminución del prejuicio y de las actitudes hostiles- a partir del contacto entre los grupos sociales en tensión, en situaciones de interacción que presenten en algún grado cuatro condiciones esenciales: similitud de estatus jerárquico entre los grupos, cooperación intergrupal (trabajan juntos en un ambiente no competitivo), objetivos comunes (dependen unos de otros para lograr las metas comunes) y apoyo de la autoridad (instituciones o reglas que enmarcan el contacto). El supuesto de la teoría es que las situaciones de interacción entre grupos distintos, en la medida en que sus miembros no se puedan evitar entre sí, exponen a sus integrantes a experiencias e impresiones acerca de los otros que modifican los estereotipos mutuos y los prejuicios asociados, facilitando así la emergencia de un conocimiento del otro mejor fundado y, por esta vía, una actitud más neutral o favorable hacia ese otro. En otras palabras, los individuos aprenden algo nuevo que modifica sus concepciones previas.

Desde entonces y hasta hoy muchos estudios han buscado evidencia que apoye o refute la teoría. Pettigrew \& Tropp (2006), realizaron un metaanálisis de 515 de estos estudios, encontrando un sólido apoyo para la hipótesis del contacto intergrupal: en el 94\% 
de los casos se encontró una correlación negativa entre contacto y prejuicio, algunas más fuertes y significativas que otras. Bajo rigurosos criterios de inclusión de los estudios que conformaron el corpus, los autores concluyeron que el contacto intergrupal no solo reduce el prejuicio mutuo, sino que estos efectos se extienden a todo el otro grupo, no solo a quienes participaron en la interacción. La generalización de los beneficios del contacto a todo el exogrupo es un logro muy apreciado por los defensores de esta teoría. Bajo las condiciones del contacto establecidas por Allport, los efectos sobre el prejuicio mutuo son aún más favorables, pero estas condiciones no son decisivas para que el efecto ocurra. Entre otras objeciones, el estudio logró refutar una no menor en importancia conceptual, esto es la dirección del efecto causal: no es el sesgo de autoselección de los más tolerantes en las situaciones de contacto lo que explica la reducción del prejuicio, sino el contacto propiamente tal. Los autores plantean que el rasgo más interesante del resultado del metaanálisis, no es tanto la fuerza de la correlación negativa entre contacto y prejuicio (en torno a -0,25), sino la amplia gama de situaciones y grupos para las cuales la teoría aplica. Tal vez porque la fuerza de la correlación es más bien moderada, sugieren que la investigación futura se focalice en determinar las condiciones negativas que neutralizan o dificultan el efecto positivo del contacto.

A pesar de la evidencia favorable a la teoría del contacto, han surgido voces en este campo que cuestionan el hecho de que se haya pasado por alto el potencial que tiene el contacto intergrupal para aumentar los prejuicios (Barlow et al., 2012; Hodson \& Hewston, 2012; Techakesari, 2015). En las conclusiones de su metaanálisis, Pettigrew y Tropp (2006) sugieren que la investigación se ha enfocado mucho en los beneficios del contacto, y que es hora de estudiar los factores y situaciones que impiden que el contacto tenga los resultados positivos en la magnitud que se esperan.

De hecho, se habla de contacto intergrupal negativo cuando al menos una de las partes se siente inconfortable con la otra, enojada o temerosa de la otra, a causa de sus actitudes y conductas en la interacción. Según Paolini, Harwood y Rubin (2010), en un contacto intergrupal negativo la percepción y descripción del otro 
en términos de su pertenencia categorial se vuelve predominante, en comparación con lo que ocurre en el contacto positivo, cuando la percepción de la pertenencia grupal del otro cede a otra en términos más personalizados. A su vez, se ha observado que las percepciones mutuas en términos de las pertenencias grupales tienden a favorecer un contacto negativo y a la generalización al exogrupo más allá de ese contacto específico, todo lo cual lleva a estos autores a sugerir que el contacto intergrupal estaría sesgado hacia el empeoramiento de las relaciones intergrupales. Por lo tanto, si una situación intergrupal contiene una mezcla de ambas experiencias positivas y negativas, estas últimas tendrán la mayor influencia en las actitudes sobre el grupo.

Putnam (2007) pone en tela de juicio la teoría del contacto, sugiriendo más bien una teoría del conflicto, pues reúne evidencia de 40 casos en Estados Unidos donde la mayor diversidad en una comunidad se asoció con una disminución de la confianza social, del altruismo y la cooperación comunitaria entre los grupos étnicos. Lo anterior ocurre incluso dentro del propio grupo étnico. Putnam sugiere que en barrios étnicamente diversos los residentes de todos los grupos tienden a "atrincherarse" y a disminuir el contacto.

Paolini, Harris y Griffin (2015) plantean que es evidente que en la gran mayoría de las situaciones experimentales, las interacciones episódicas con miembros del exogrupo, al menos en el corto plazo, causan un aumento en los niveles de ansiedad, lo que actúa como un motivo para evitar el contacto y tiene efectos perjudiciales sobre los juicios intergrupales. No obstante, el contacto intergrupal, junto con ofrecer la oportunidad de aprender a estar ansioso hacia el exogrupo, también ofrece la posibilidad de revisar esas ansiedades. Como consecuencia de ello, las respuestas hacia el exogrupo pueden cambiar, para bien o para mal, con el tiempo, a través de un proceso de aprendizaje. Los individuos acumulan e integran sus experiencias crónicas con el exogrupo, disponiéndose para nuevas experiencias de contacto y moldeando, para bien o para mal, sus respuestas episódicas al exogrupo.

Algunos estudios recientes se han inspirado en la teoría del contacto para analizar sus efectos en la escuela, entre estudiantes de 
diferentes etnias u orígenes nacionales, normalmente inmigrantes. Un autor en particular, Jan Janmaat $(2012,2014,2015)$, ha llevado a cabo varios estudios para averiguar si la mixtura étnico racial en el aula favorece o no la tolerancia social y otras actitudes afines a la cohesión social. En general, sus resultados muestran que dicha mixtura en la escuela no tiene los mismos efectos en todas las actitudes estudiadas ni en todos los países. Los factores específicos de cada uno dan forma al efecto del contacto en un grado significativo.

En un estudio reciente Janmaat (2015) analiza si ciertas actitudes cívicas de los estudiantes ingleses nativos (blancos) son o no influidas por la mixtura étnica en la sala de clases. Además de la inclusividad en relación con los inmigrantes, incluyó la confianza hacia otras personas de la misma edad como variables dependientes. El autor quería contrastar dos perspectivas teóricas opuestas respecto de los efectos de la diversidad. La teoría del contacto predice resultados positivos sobre ambas actitudes en observación, pero la perspectiva del conflicto predice un aumento de la desconfianza en contextos de diversidad étnica. Encontró al principio un vínculo positivo entre la diversidad y la actitud inclusiva de los estudiantes blancos, pero una vez que se añadieron controles para el género, el origen social y valores previos de inclusividad y confianza (de mediciones anteriores con el mismo instrumento) el "efecto" positivo de la diversidad desapareció. Este efecto positivo inicial de la diversidad solo reflejaba un efecto de autoselección, correspondiente a una sobrerrepresentación en las aulas diversas de los estudiantes blancos con ciertas características previas, como altos niveles de inclusividad y otros antecedentes específicos. Por otra parte, los efectos de la diversidad étnica sobre la confianza fueron negativos, incluso incorporando varios controles, lo cual va en apoyo de la perspectiva del conflicto.

La literatura en torno a la teoría del contacto ha trabajado con grupos de distinta naturaleza -especialmente étnicos e inmigrantes-, pero no con grupos diferenciados por el NSE. En Chile, este tipo de diferenciación social es relevante. Por otra parte, no se cuenta con evidencia del impacto de la segregación/inclusión social de las escuelas sobre dimensiones actitudinales relacionadas con la formación ciudadana. Este artículo se propone indagar en este dominio y 
aportar alguna evidencia de lo que ocurre en estos espacios escolares con ciertas actitudes y comportamientos como la tolerancia social, la confianza en la gente, la convivencia entre pares, en el entendido de que estas actitudes y comportamientos podrían ser positivos o negativos en situaciones de contacto intergrupal en la escuela. La teoría del contacto intergrupal, o la del conflicto, son solo un apoyo en esta exploración; no se formulan hipótesis formales derivadas de ellas. De las muchas actitudes y conductas cívicas estudiadas en ICCS 2009, el presente estudio seleccionó siete (ver descripción más adelante) no solo porque son relevantes en la formación ciudadana, sino porque serían sensibles a la condición de convivir entre iguales o personas diferentes en términos de estatus socioeconómico en una escuela. ¿Son estas actitudes más positivas o negativas en espacios de mixtura social que en espacios escolares segregados? Otras variables actitudinales abordadas en ICCS 2009, como la confianza en las instituciones o la intención de participación electoral, no se escogieron porque no tendrían una relación directa con el carácter segregado o inclusivo de la escuela. Por cierto, para realizar la comparación este estudio supone la existencia de escuelas inclusivas en medio de la realidad escolar segregada de Chile. Como se verá, tales escuelas existen: su puesta en evidencia representa un hallazgo de este estudio.

\section{Método}

Se utilizaron las bases de datos del estudio ICCS 2009, disponibles en el sitio web de la institución organizadora. La muestra de Chile corresponde a 5.192 estudiantes de $8^{\circ}$ año básico pertenecientes a 177 establecimientos educacionales de todo el país (Ministerio de Educación, 2010). En dicho estudio, los establecimientos fueron elegidos a través de un muestreo aleatorio estratificado, con probabilidad proporcional al tamaño de la escuela. Luego, en cada establecimiento seleccionado se eligió al azar un curso completo de $8^{\circ}$ básico, todos los profesores de ese grado (con un máximo de 20) y el director o directora.

ICCS 2009 consistió en la aplicación de dos instrumentos comunes a todos los países: una prueba de conocimientos cívicos y un cuestionario para medir percepciones, actitudes y comportamientos 
relevantes para la formación ciudadana. Adicionalmente, los estudiantes respondieron un módulo regional, consistente en una prueba de conocimientos y un cuestionario de actitudes complementarias que recogió información acerca de conocimientos y actitudes cívicos relevantes para sus regiones o continentes. Así, el cuestionario latinoamericano de actitudes incluyó, por ejemplo, el tema de la dictadura como forma de gobierno y la disposición a convivir en el mismo barrio con diferentes tipos de personas; temas ausentes en los otros módulos regionales.

El procedimiento metodológico de la presente investigación siguió dos etapas. En la primera, se procedió a describir y clasificar a los establecimientos de acuerdo con el tipo de composición social en su interior, es decir, escuelas que agrupan a estudiantes socialmente desaventajados, o a estudiantes socialmente aventajados, o de diversas condiciones socioeconómicas. El nivel socioeconómico (NSE) de los estudiantes corresponde al índice elaborado por ICCS 2009 de estatus económico, social y cultural, el cual integra información del nivel educacional de los padres, su nivel ocupacional y la cantidad de libros en el hogar (Schultz, Ainley \& Fraillon, 2011). Así se calculó el promedio de este índice de cada escuela de la muestra y su diferencia, positiva o negativa, con el promedio nacional. De acuerdo con la prueba $t$ de Student, las escuelas con promedios significativamente más bajos o más altos que el nacional se consideraron segregadas desaventajadas o segregadas aventajadas, respectivamente, en tanto que aquellas cuyos promedios no eran significativamente distintos del nacional (estaban en el intervalo $+-1,96$ ) se consideraron mixtas o inclusivas. Este método de clasificación es reportado en el informe técnico de otro estudio evaluativo internacional (OECD, 2009) y usado en el informe de resultados PISA 2012 como un indicador de equidad (OECD, 2013).

Ahora bien, las escuelas cuyo NSE promedio no difiere significativamente del promedio nacional pueden componerse, en principio, de estudiantes muy cercanos al NSE promedio nacional y/o de estudiantes de diferentes NSE, de tal modo que el promedio de ellos se acerque al promedio nacional. Así, luego de identificadas las escuelas de los tres grupos, se revisó la composición social interna 
de cada una, en términos de cuáles y cuántos quintiles de NSE de los estudiantes estaban presentes, para validar la clasificación anterior. Para la determinación de los quintiles nacionales de NSE se calcularon los percentiles 20, 40, 60 y 80 del índice ICCS de estatus económico social y cultural, integrando los pesos de los estudiantes. La revisión verificó que el grupo de escuelas inclusivas se compone de estudiantes de, al menos, cuatro quintiles de NSE, en proporciones sino estrictamente homogéneas, razonablemente repartidas, como lo revela el Cuadro 1. 


\begin{tabular}{|c|c|c|c|c|c|c|c|c|c|c|c|c|}
\hline \multirow{7}{*}{ 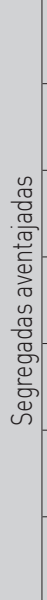 } & $\begin{array}{l}\overrightarrow{\widetilde{0}} \\
\text { 믐 }\end{array}$ & $\gtrsim$ & m & ল & లి & 寸 & $\approx$ & $\simeq$ & $\approx$ & $\approx$ & $\widetilde{m}$ & $\approx$ \\
\hline & $\stackrel{18}{0}$ & 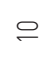 & 이 & 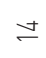 & $\curvearrowright$ & $\approx$ & $\approx$ & 으 & $\simeq$ & $\stackrel{2}{\circ}$ & $\approx$ & $\approx$ \\
\hline & む & 0 & 으 & $\simeq$ & 으 & $\simeq$ & - & 0 & $\infty$ & $m$ & $a$ & ما \\
\hline & 乃 & $m$ & $=$ & $\checkmark$ & $m$ & 0 & 0 & $\sim$ & $m$ & - & - & 0 \\
\hline & ชี & $m$ & $\sim$ & $m$ & $\sim$ & $m$ & 0 & 0 & 0 & 0 & 0 & 0 \\
\hline & $\bar{\sigma}$ & $\sim$ & m & - & - & 0 & 0 & 0 & 0 & 0 & 0 & 0 \\
\hline & نّ & ᄋ & $\stackrel{\mathscr{I}}{\subseteq}$ & $\stackrel{\stackrel{\rho}{\ominus}}{\circ}$ & ఫ্ & $\stackrel{o}{\subseteq}$ & 㑒 & \begin{tabular}{l}
$\infty$ \\
$o$ \\
$o$ \\
\hdashline
\end{tabular} & $\begin{array}{l}\text { oे } \\
\text { o }\end{array}$ & 영 & $\bar{\sigma}$ & 응 \\
\hline \multirow{7}{*}{ 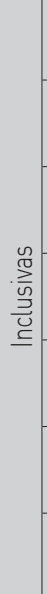 } & $\begin{array}{l}\overrightarrow{\widetilde{T}} \\
\stackrel{\overrightarrow{0}}{\circ}\end{array}$ & 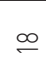 & $m$ & ল & లి & $\infty$ & 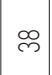 & $m$ & $\bar{m}$ & ঐ & $\approx$ & $\approx$ \\
\hline & $\stackrel{2}{0}$ & $\sim$ & | & $\sim$ & $\checkmark$ & مـ & $\sim$ & - & $\sim$ & ما & $\infty$ & $\checkmark$ \\
\hline & む & $m$ & 으 & $\sigma$ & 으 & $\approx$ & $\circ$ & $\infty$ & 0 & $m$ & 0 & $=$ \\
\hline & \% & $\sim$ & $a$ & 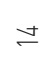 & $\circ$ & $\circ$ & $\infty$ & $=$ & $a$ & $\infty$ & $\sim$ & م \\
\hline & ชี & م & $\sim$ & $a$ & $\sim$ & 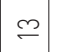 & $\simeq$ & $\infty$ & $a$ & $\infty$ & $a$ & - \\
\hline & б & 0 & $\sim$ & $\checkmark$ & م & $m$ & - & مـ & ما & ما & - & - \\
\hline & نّ & $\bar{\varnothing}$ & $\bar{\varnothing}$ & $\stackrel{\infty}{\circ}$ & 立 & $\Xi$ & $\stackrel{\stackrel{\sim}{\sigma}}{\stackrel{\sigma}{\sigma}}$ & 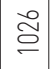 & $\stackrel{\infty}{\Xi}$ & ప్ & ஜ్ర & 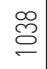 \\
\hline \multirow{7}{*}{ 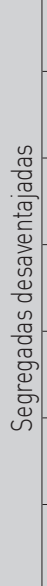 } & $\begin{array}{l}\overrightarrow{\widetilde{T}} \\
\stackrel{0}{\circ}\end{array}$ & $\bar{v}$ & $\curvearrowright$ & $\simeq$ & $\stackrel{20}{\circ}$ & $\stackrel{\sim}{\sim}$ & $\approx$ & 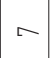 & 0 & $\tilde{m}$ & 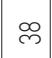 & $\widetilde{m}$ \\
\hline & $\stackrel{2}{0}$ & $\sim$ & $\sim$ & - & - & 0 & 0 & 0 & 0 & 0 & $\sim$ & - \\
\hline & d & مـ & $\sim$ & 0 & $\sim$ & - & 0 & - & 0 & 0 & $m$ & $\checkmark$ \\
\hline & 8 & - & $m$ & - & - & - & 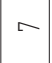 & 0 & 0 & 0 & ما & - \\
\hline & ర్ & $m$ & $\checkmark$ & - & $m$ & L & $\checkmark$ & $\sim$ & - & $=$ & $a$ & $a$ \\
\hline & $\bar{\sigma}$ & 으 & $a$ & $\infty$ & $\infty$ & $\infty$ & $\infty$ & $\checkmark$ & ما & \pm & $\stackrel{\sigma}{\circ}$ & $\simeq$ \\
\hline & نّ & ᄋ응 & $\stackrel{\infty}{\circ}$ & $\stackrel{\circ}{\circ}$ & 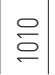 & $\bar{\sigma}$ & $\stackrel{\simeq}{\check{\sigma}}$ & $\stackrel{m}{\sigma}$ & 음 & ఠ్ర & $\bar{\sigma}$ & $\underset{\Xi}{\Xi}$ \\
\hline
\end{tabular}


En la segunda etapa, se procedió a analizar la relación entre los tres grupos de composición social y siete variables dependientes actitudinales y comportamentales de las estudiantes, todas disponibles en las bases de datos de ICCS 2009.

\section{a. Actitud hacia la diversidad social en el vecindario}

La pregunta 6 del módulo latinoamericano interrogó a los estudiantes acerca de cómo reaccionarían ("me gustaría", "no me importaría", "me disgustaría") frente a tener vecinos de diversas poblaciones, así como vecinos con estilos de vida particulares o con discapacidades. En la pregunta se presentaron 10 alternativas de vecinos diferentes ("gente de color de piel distinta a la tuya"; "gente de clase social distinta a la tuya"; "gente de religión distinta a la tuya"; "homosexuales o lesbianas"; "gente que viene de otra región del país"; "personas con discapacidades físicas"; "personas con discapacidades mentales"; "gente de una nacionalidad distinta a la tuya"; "personas con sida"; "personas de origen indígena") que fueron utilizadas en ICCS 2009 para construir una escala numérica ${ }^{9}$, cuyos puntajes más altos corresponden a una mayor aceptación de la diversidad.

b. Actitud hacia la convivencia con gente de otra clase social en el vecindario

Uno de los ítems de la pregunta 6 recién mencionada es "gente de clase social distinta a la tuya". La escala aludida en el punto anterior, al integrar las respuestas de los estudiantes frente a distintos objetos de actitud (por ejemplo, discapacitados, etnias diferentes, etc.), invisibiliza la información de las actitudes específicas hacia los diferentes objetos sociales, las cuales podrían ser positivas hacia unos y negativas hacia otros, siendo neutralizadas en la escala. "Gente de clase social distinta a la tuya" alude a un objeto de actitud relacionado de manera más pertinente con los propósitos del estudio, en el sentido de que la convivencia escolar en ambientes de mixtura social podría estar más asociada a la tolerancia hacia la gente de una clase social distinta, que a la tolerancia hacia otros objetos de actitud,

9 Se usó el modelo de respuesta al ítem para escalar los ítems y se realizaron análisis factoriales confirmatorios. Todas las escalas se estandarizaron con un promedio de 50 y una desviación estándar de 10. 
como "homosexuales y lesbianas", "personas de origen indígena" y otros. De las tres opciones de respuesta frente al ítem "gente de clase social distinta a la tuya" se tomaron en cuenta las opciones: "me gustaría" y "no me importaría", resultando en una variable dependiente dicotómica: indiferente o positiva. Pese a que ambas opciones no son particularmente contradictorias entre sí, implican una gradación significativa de la actitud de aceptación del otro. Hubiese sido interesante incluir en el análisis a la tercera opción de respuesta "me disgustaría", preferida solo por el 5\% de los estudiantes, pero fue excluida para permitir un análisis de regresión logística binaria ${ }^{10}$.

\section{c. Confianza en la gente}

Se les preguntó a los estudiantes: "¿cuánto confías en cada uno de los siguientes grupos o instituciones?". El listado incluía 12 entidades, pero este estudio escogió solo una de ellas: "las personas en general". Las opciones de respuesta eran "completamente", "bastante", "un poco", "nada". Se agruparon las respuestas de las dos primeras opciones y de las dos segundas, resultando así una variable dependiente dicotómica, confianza en la gente: no o sí.

\section{d. Sentimiento de empatía hacia los compañeros}

La pregunta 9 del módulo latinoamericano les presentó a los estudiantes 10 ítems acerca de diversas situaciones que ellos podrían presenciar en la escuela y que involucraban a compañeros de clase ("un compañero de clase se cae y se hace daño"; "a un compañero de clase le pegan"; "regañan injustamente a un compañero"; "castigan injustamente a un compañero"; "a un compañero de clase le roban algo"; "se burlan de un compañero"; "insultan a un compañero"; "un compañero de clases está muy triste"; "un compañero saca malas calificaciones"; "un compañero no tiene con quién jugar"). Se les pidió a los estudiantes que expresaran cómo se sentían frente a los acontecimientos representados ("creo que es divertido", "no me

10 Lamentablemente, el software IDB Analyzer, utilizado para resguardar la construcción de las sintaxis de SPSS de acuerdo con los requerimientos metodológicos de ICCS 2009, no permite más de dos categorías de las variables dependientes en el análisis de regresión logística. En todo caso, no se observa una preferencia marcada de alguno de los tres grupos de escuelas por la opción "me disgustaría" (ver en Anexo cuadro i). 
importa", "me molesta"). Con las respuestas a los 10 ítems ICCS 2009 se construyó otra escala, cuyas puntuaciones altas expresaban un mayor grado de empatía hacia los compañeros.

e. Rechazo a ser incluido en un grupo

El cuestionario latinoamericano incluyó una pregunta para saber si los estudiantes habían experimentado agresión verbal o física en la escuela durante el mes anterior a la encuesta, uno de cuyos ítems decía "alguien te rechazó y no te dejó estar en su grupo". Los estudiantes reportaron con qué frecuencia ("nunca", "solamente una vez", "dos a cuatro veces", "cinco veces o más") les sucedió aquello. Se agruparon las opciones de respuesta en dos alternativas: "nunca", por un lado y el resto de las opciones originales por otro. El resultado fue otra variable dependiente dicotómica: nunca o sí.

\section{f. Ser objeto de insultos}

Otro ítem de la pregunta anterior decía: "alguien en tu colegio te insultó". Los estudiantes reportaron con qué frecuencia ("nunca", "solamente una vez", "dos a cuatro veces", "cinco veces o más") les sucedió aquello. Se agruparon las opciones de respuesta en dos alternativas: "nunca", por un lado y el resto de las opciones originales por otro. El resultado fue otra variable dependiente dicotómica: nunca o sí.

g. Ser objeto de apodos ofensivos

Otro ítem de la misma pregunta, decía: "un compañero te llamó por un apodo ofensivo". Se agruparon las opciones de respuesta en dos alternativas: "nunca", por un lado y el resto de las opciones originales por otro, resultando otra variable dependiente dicotómica: nunca o sí.

Ninguna de las tres variables dependientes derivadas de la pregunta por agresiones verbales o físicas experimentadas en la escuela aludían al NSE como motivo de la agresión; tampoco las de empatía se referían al NSE de los estudiantes. Pero si estas dificultades ocurrieran en las escuelas inclusivas más que en las segregadas cabría pensar, si otras condiciones se mantienen constantes, que la mezcla social apoya la hipótesis del conflicto más que la del contacto. 
Como variables independientes se incluyó, el tipo de composición social de la escuela (variable tricotómica con las escuelas inclusivas como grupo de referencia), al género del estudiante (hombres como grupo de referencia) y a su NSE (variable numérica). Los informes de resultados entregados por ICCS 2009 mostraban que en general el género era un factor de diferenciación de varias actitudes cívicas de los estudiantes, de modo que era necesario tenerlo controlado. Con respecto del NSE del estudiante ${ }^{11}$, también presente en los informes de resultados, se optó por controlar su efecto eventual, considerando que al interior de cada uno de los tres tipos de escuela subsiste cierta variabilidad del NSE de sus estudiantes.

El análisis utilizó regresiones, lineales y logísticas binarias. El uso de las regresiones aquí no tiene como fin ajustar un buen modelo que prediga las variables dependientes, sino analizar la relación de los tres grupos de escuelas con las variables dependientes, su significación estadística, controlando por el género y el NSE de los estudiantes. Para las variables dependientes que son escalas numéricas se usaron regresiones lineales; en tanto que para las categoriales dicotómicas, regresiones logísticas. El análisis de regresión logística es similar a un modelo de regresión lineal, pero adaptado a una variable dependiente categorial, dicotómica en este caso (0 y 1 en el lenguaje del sistema, donde la categoría de referencia es 0 ). Si bien aquí esta subutilizado, este tipo de análisis resulta útil para determinar cuál o cuáles variables independientes están asociadas a la probabilidad de que la variable dependiente sea 1, su significación estadística, y cuán grande es esta probabilidad (odds ratios o razones de probabilidad). Las categorías que asumen el valor 1 en las variables dependientes de este estudio son: "me gustaría" (convivir con gente de otra clase social en el vecindario), "sí confío en la gente", "sí he sido rechazado" "sí he sido insultado", "sí he recibido apodos ofensivos".

Todos los análisis se realizaron de acuerdo con los procedimientos estadísticos establecidos en el reporte técnico del estudio ICCS 2009 (Schulz, Ainley \& Fraillon, 2011). Particularmente importante es el procedimiento que exige que el error estándar se calcule con gran

11 Corresponde al índice estatus económico, social y cultural elaborado por ICCS 2009. 
precisión, mediante operaciones adicionales a las que entregan por defecto los paquetes estadísticos. El cálculo del error estándar de cualquier parámetro poblacional fue replicado 75 veces siguiendo el método Jacknife. Mediante este procedimiento de replicación se neutraliza la subestimación del error estándar asociada a la violación del supuesto de independencia de las observaciones, que caracteriza a las muestras de estudiantes anidados en escuelas. Otra precaución en los análisis es la consideración de los pesos que deben ser asociados a cada estudiante y a cada escuela, de acuerdo con su estrato de pertenencia.

\section{Resultados}

En primer lugar se presentan algunos resultados descriptivos de la primera etapa del estudio, es decir, de la clasificación de los establecimientos según el tipo de composición social de sus alumnos. El Cuadro 2 muestra las frecuencias relativas de estudiantes en cada tipo de escuela; se agrega el puntaje en la prueba de conocimientos cívicos como una referencia. La magnitud de los estudiantes en el grupo de escuelas inclusivas es indicativa del nivel de inclusión social y/o segregación del sistema escolar: en Chile el 70\% estudia en escuelas segregadas. Obsérvese que los puntajes de los tres tipos de escuelas en la prueba de conocimientos cívicos son coherentes con la relación típica entre el NSE y el logro académico; a mayor nivel, mayor puntaje.

Cuadro 2. Distribución de los estudiantes en escuelas inclusivas y segregadas len porcentaje), género y puntaje promedio de logro en conocimiento cívico

\begin{tabular}{|c|c|c|c|}
\hline Composición social de la escuela & Porcentaje & Género & Puntaje promedio \\
\hline \multirow{2}{*}{ Segregada desaventajada } & \multirow{2}{*}{39,2} & 49,8 hombres & \multirow{2}{*}{445,7} \\
\hline & & 50,2 mujeres & \\
\hline \multirow{2}{*}{ Inclusiva } & \multirow{2}{*}{30,6} & 51,8 hombres & \multirow{2}{*}{476} \\
\hline & & 48,2 mujeres & \\
\hline \multirow{2}{*}{ Segregada aventajada } & \multirow{2}{*}{30,2} & 45,7 hombres & \multirow{2}{*}{539,3} \\
\hline & & 54,3 mujeres & \\
\hline
\end{tabular}

En cuanto a la composición interna de cada uno de estos tres grupos de establecimientos, puede observarse que se encuentran estudiantes de los cinco quintiles de NSE en proporciones diversas de 
acuerdo con el tipo de inclusión/exclusión social que le es característico (ver Cuadro 3). En los segregados desaventajados, el quintil 1 es el mayoritario y es seguido del quintil 2, en tanto que los quintiles 3 y 4 son minoritarios; sorprendentemente hay estudiantes del quintil 5, donde no se esperaría ninguno. En el grupo de los segregados aventajados se invierten estas proporciones, predominando el quintil 5 , seguido del quintil 4 y luego los otros, con una presencia escasísima del quintil 1. El grupo de los establecimientos con mixtura social tiene una distribución semejante a la normal.

Cuadro 3. Distribución del alumnado por quintiles de NSE en los tres grupos de establecimientos (en porcentaje)

\begin{tabular}{l|c|c|c|c|c|c}
\hline & \multicolumn{7}{|c}{ Quintiles NSE } \\
\hline Tipo escuela & Q1 & Q2 & Q3 & Q4 & Q5 & Total \\
\hline Segregadas desaventajadas & 41,7 & 27,5 & 16,9 & 10,2 & 3,7 & 100 \\
\hline Inclusivas & 10,9 & 22,7 & 28,9 & 24,8 & 12,7 & 100 \\
\hline Segregadas aventajadas & 1,8 & 7,2 & 14,3 & 27,8 & 49 & 100 \\
\hline
\end{tabular}

La segunda etapa del estudio arroja los siguientes resultados, por cada variable dependiente.

\section{a. Actitud hacia la diversidad social en el vecindario}

Los coeficientes de regresión B tienen signo distinto para ambos tipos de escuelas segregadas. El signo negativo asociado a las segregadas pobres indica que, con respecto de las inclusivas, son menos tolerantes. En cambio, las segregadas aventajadas son más tolerantes con respecto a las inclusivas. Pero ambos coeficientes son no significativos. Los b.t del cuadro (valores $t$ de Student) fuera del rango +- 1,96; otorgan significación estadística a los coeficientes de regresión respectivos. Este solo es el caso de la variable género: las mujeres son más aceptadoras de la diversidad social en el vecindario respecto de los hombres. 
Cuadro 4. Regresión lineal sobre la variable "Actitud hacia la diversidad social en el vecindario"

\begin{tabular}{l|c|c|c}
\hline & B & b.se & b.t \\
\hline (Constant) & 51,67 & 0,19 & 268,12 \\
\hline NSE del estudiante & $-0,05$ & 0,21 & $-0,22$ \\
\hline Segregada desaventajada & $-0,28$ & 0,36 & $-0,78$ \\
\hline Segregada aventajada & 0,04 & 0,29 & 0,14 \\
\hline Género: mujer & 1,08 & 0,17 & 6,2 \\
\hline
\end{tabular}

b. Actitud hacia la convivencia con gente de otra clase social en el vecindario

Dado su carácter categorial, la pregunta por el grado de aceptación a convivir con gente de otra clase social en el vecindario se analizó mediante una regresión logística. Los coeficientes de los dos tipos de escuelas segregadas tienen signo negativo, lo que indica que, en comparación con las escuelas inclusivas, están más cerca de la indiferencia que de la aceptación; sin embargo, solo las escuelas segregadas ricas son más indiferentes de manera estadísticamente significativa, aunque con una probabilidad baja, según el valor b.exp. Los coeficientes asociados al NSE del estudiante y al género, no son significativos. Esta es la única variable dependiente respecto de la cual el género no resultó significativo.

Cuadro 5. Regresión logística sobre la variable “Aceptación de gente de otra clase social en el vecindario"

\begin{tabular}{l|c|c|c|c}
\hline & B & b.se & b.sig & b.exp \\
\hline CONSTANT & $-0,43$ & 0,08 & 0 & 0,648 \\
\hline NSE del estudiante & 0,04 & 0,04 & 0,363 & 1,041 \\
\hline Segregada desaventajada & $-0,07$ & 0,12 & 0,562 & 0,933 \\
\hline Segregada aventajada & $-0,23$ & 0,09 & 0,012 & 0,796 \\
\hline Género: mujer & 0,02 & 0,09 & 0,807 & 1,022 \\
\hline
\end{tabular}

c. Confianza en la gente

Aquí también se usó una regresión logística. Solo es estadísticamente significativa la variable género, el signo negativo indica que las estudiantes tienen más probabilidad de no tener confianza en la gente, en relación con sus pares hombres, aunque esa probabilidad es 
modesta. Las escuelas segregadas, respecto de las inclusivas, presentan coeficientes insignificantes.

Cuadro 6. Regresión logística sobre la variable "Confianza en la gente"

\begin{tabular}{l|l|l|l|l}
\hline & B & b.se & b.sig & b.exp \\
\hline CONSTANT & 0,16 & 0,06 & 0,01 & 1,169 \\
\hline NSE del estudiante & $-0,02$ & 0,04 & 0,592 & 0,98 \\
\hline Segregada desaventajada & $-0,01$ & 0,1 & 0,94 & 0,992 \\
\hline Segregada aventajada & 0,06 & 0,08 & 0,512 & 1,057 \\
\hline Género: mujer & $-0,19$ & 0,07 & 0,011 & 0,827 \\
\hline
\end{tabular}

\section{d. Empatía hacia los compañeros}

A pesar de que todas las variables independientes predicen la empatía, no todas son significativas. Ambos tipos de escuelas segregadas predicen mejor la empatía que las inclusivas, pero solo las aventajadas lo hacen de manera significativa. El NSE del estudiante no es significativo, pero sí lo es el género: las mujeres son más empáticas que los hombres.

Cuadro 7. Regresión lineal sobre la variable "Empatía hacia los compañeros"

\begin{tabular}{l|c|c|c}
\hline & B & b.se & b.t \\
\hline (Constant) & 48,76 & 0,22 & 220,19 \\
\hline NSE del estudiante & 0,15 & 0,2 & 0,73 \\
\hline Segregada desaventajada & 0,1 & 0,37 & 0,26 \\
\hline Segregada aventajada & 1,02 & 0,35 & 2,9 \\
\hline Género: mujer & 2,25 & 0,15 & 15,21 \\
\hline
\end{tabular}

e. Rechazo a ser incluido en un grupo

Esta variable dependiente se analizó mediante una regresión logística. El coeficiente positivo de las escuelas segregadas pobres indica que sus estudiantes sí han tenido más rechazo en relación con las inclusivas, pero no es significativo. Solo dos coeficientes tienen significación estadística y ambos son negativos; los estudiantes de las escuelas segregadas aventajadas, respecto de las inclusivas, presentan una mayor probabilidad de nunca haber sido rechazados. Lo mismo ocurre con las mujeres respecto de los hombres. 
Cuadro 8. Regresión logística sobre la variable "Rechazo a ser incluido en un grupo"

\begin{tabular}{l|c|c|c|c}
\hline & B & b.se & b.sig & b.exp \\
\hline CONSTANT & $-0,75$ & 0,08 & 0 & 0,472 \\
\hline NSE del estudiante & $-0,06$ & 0,04 & 0,155 & 0,942 \\
\hline Segregada desaventajada & 0,03 & 0,12 & 0,811 & 1,03 \\
\hline Segregada aventajada & $-0,31$ & 0,11 & 0,004 & 0,734 \\
\hline Género: mujer & $-0,19$ & 0,08 & 0,012 & 0,827 \\
\hline
\end{tabular}

f. Ser objeto de insultos

Esta variable dependiente presenta el mismo patrón de resultados que la anterior. La regresión logística muestra que solo dos coeficientes tienen significación estadística y ambos son negativos; esto significa que los estudiantes de las escuelas segregadas aventajadas, respecto de las inclusivas, están más cerca de la probabilidad de nunca haber sido insultados en el último mes. Lo mismo ocurre en el caso de las mujeres respecto de los hombres.

Cuadro 9. Regresión logística sobre la variable "Ser objeto de insultos"

\begin{tabular}{l|c|c|c|c}
\hline & B & b.se & b.sig & b.exp \\
\hline CONSTANT & 1,02 & 0,08 & 0 & 2,779 \\
\hline NSE del estudiante & $-0,01$ & 0,04 & 0,769 & 0,989 \\
\hline Segregada desaventajada & 0,08 & 0,11 & 0,476 & 1,083 \\
\hline Segregada aventajada & $-0,27$ & 0,1 & 0,004 & 0,76 \\
\hline Género: mujer & $-0,65$ & 0,08 & 0 & 0,522 \\
\hline
\end{tabular}

g. Ser objeto de apodos ofensivos

La regresión logística muestra tres coeficientes con significación estadística y los tres son negativos. Los estudiantes de las escuelas segregadas aventajadas, respecto de las inclusivas, están más cerca de la probabilidad de nunca haber recibido un apodo ofensivo en el último mes. Lo mismo para el caso de las mujeres respecto de los hombres y de los estudiantes de NSE superiores. Es la única variable dependiente respecto de la cual el NSE del estudiante tiene una relación significativa independiente. Las segregadas pobres, en cambio, tienen mayor probabilidad de haber recibido apodos ofensivos que las inclusivas, pero sin significación. 
Cuadro 10. Regresión logística sobre la variable "Ser objeto de apodos ofensivos"

\begin{tabular}{l|c|c|c|c}
\hline & B & b.se & b.sig & b.exp \\
\hline CONSTANT & 0,5 & 0,08 & 0 & 1,651 \\
\hline NSE del estudiante & $-0,11$ & 0,04 & 0,012 & 0,897 \\
\hline Segregada desaventajada & 0,15 & 0,11 & 0,189 & 1,16 \\
\hline Segregada aventajada & $-0,26$ & 0,1 & 0,008 & 0,771 \\
\hline Género: mujer & $-0,49$ & 0,06 & 0 & 0,612 \\
\hline
\end{tabular}

Finalmente, una nota metodológica. Si no se hubiese calculado con precisión el error estándar, según se explicó en la descripción del método, otros serían los resultados. Las mismas regresiones, ejecutadas solo con las operaciones por defecto del SPSS, dan significación estadística a casi todos los coeficientes.

\section{Discusión y conclusiones}

En general, los resultados muestran que los estudiantes de las escuelas inclusivas tienen disposiciones actitudinales distintas de los de las segregadas aventajadas en la mayoría de las variables dependientes en estudio. A su vez, los primeros tienden a parecerse a los estudiantes de las segregadas pobres respecto de varias actitudes. Dadas las variables de control introducidas, la composición social de las escuelas genera ambientes relativamente diferenciados en relación con las variables actitudinales estudiadas. Dicho esto, las diferencias actitudinales entre hombres y mujeres son más claras que las asociadas a la composición social de las escuelas. En cambio el NSE del estudiante no mostró una relación independiente de la composición social de las escuelas y del género en la mayoría de las variables dependientes.

Intuitivamente se esperaba un resultado más claro a favor de las escuelas inclusivas en lo relativo a la tolerancia frente a la convivencia con gente de otra clase social en el vecindario: sí son más tolerantes que sus pares de escuelas segregadas, pero solo de las aventajadas de manera significativa. Aunque modesto, se puede decir que este resultado va en la línea de lo esperado con las políticas de inclusión. Sin embargo, no se puede interpretar que el contacto con otros socialmente diferentes en las escuelas inclusivas genere mayor tolerancia hacia personas de clases sociales diferentes. Aún en el caso 
de que las diferencias con las segregadas pobres fueran significativas, es posible que el público escolar que asiste a estas escuelas mixtas, llegue con una experiencia anterior de mayor convivencia con gente de diferentes clases sociales. Nada se sabe de los procesos de constitución de las escuelas inclusivas: podrían ser una formación natural en ciertas comunas socialmente heterogéneas.

En cuanto a la empatía hacia los compañeros, los estudiantes de las escuelas inclusivas son menos empáticos que sus pares de escuelas segregadas aventajadas. En la misma línea, tanto la experiencia de rechazo a estar en un grupo, como ser insultado o recibir apodos ofensivos, son significativamente más probables en las inclusivas que en las segregadas aventajadas. ¿Alude este resultado a la presencia de una mayor conflictividad en las escuelas inclusivas, atribuible a tensiones relacionadas con el NSE? No parece ser el caso, pues las segregadas pobres se comportan de modo similar a las inclusivas. En las primeras habría entonces tanta conflictividad como en las segundas, aunque tal vez por motivos diferentes. Las preguntas del cuestionario ICCS 2009 no especifican las causas o motivos de las experiencias de agresión vividas en la escuela, por lo que no podemos saber si las razones de la mayor conflictividad que existe en las segregadas pobres y en las inclusivas, respecto de las segregadas aventajadas, son iguales o diferentes. Por de pronto, las preguntas del cuestionario no aluden al NSE de los estudiantes como objeto de actitud, tema que también puede estar influyendo en los resultados encontrados y que sería conveniente subsanar en estudios próximos.

En cuanto a la confianza en la gente, los antecedentes teóricos y empíricos expuestos predecirían una mayor desconfianza en las escuelas inclusivas; en contextos segregados la confianza es más probable que en contextos mixtos. Pero en estas escuelas los estudiantes no muestran diferencias con sus pares de las escuelas segregadas de ambos tipos. La pregunta del cuestionario hablaba de "confianza en las personas"; si hubiese sido "confianza en los compañeros", tal vez los resultados hubiesen sido distintos.

Llama la atención la poca diferenciación encontrada entre los estudiantes de escuelas inclusivas y los de escuelas segregadas pobres, 
respecto de las actitudes observadas. Da la impresión de que en las escuelas inclusivas los estudiantes no se percibieran como miembros de grupos sociales distintos y, por tanto, no existiera contacto intergrupal en términos de esta teoría. Tal vez la composición social de ambos tipos de escuelas no es demasiado diferente en lo social y cultural. Tómese en cuenta que no se está en presencia de una segregación social entre actores bien polarizados, como entre blancos y negros, o nativos e inmigrantes; acá se trata de individuos ubicados en distintos niveles de un continuo de NSE. Las diferencias de perfil sociocultural entre los quintiles de NSE contiguos serían más bien pequeñas, no así entre los extremos. Esta observación no es válida en cuanto al rendimiento académico, donde cada quintil suele tener una diferencia significativa con los contiguos en los puntajes de las pruebas de logro; tal vez tampoco es válida para cualquier objeto de actitud.

Entonces, una interpretación posible de estos resultados es que a las escuelas inclusivas les faltó una presencia mayor de estudiantes del quintil 5 de NSE. Este quintil tiene un perfil sociocultural claramente delineado y distinto del resto. Complementariamente, en las escuelas inclusivas el quintil 3 tiene una presencia preponderante, quintil que tendría un perfil más cercano a los quintiles inferiores que a los superiores. Ejercicios de análisis estadísticos hechos con posterioridad al desarrollo de este estudio dan sustento a la interpretación anterior; respecto del quintil 1 de NSE de la escuela, el único quintil significativamente diferente en varios dominios actitudinales observados fue el quintil 5. Así, en las escuelas inclusivas de este estudio la composición social en su interior no alcanzaría a generar procesos de polarización sociocognitiva en los estudiantes, cuya resolución los condujera a cambios actitudinales respecto de sus pares de escuelas segregadas pobres.

Las escuelas con mixtura social ¿desarrollan procesos educativos intencionalmente inclusivos? Si la constitución de este tipo de establecimientos fuera consecuencia de proyectos educativos inclusivos de sostenedores y comunidades bien intencionados, se esperaría un desarrollo positivo más acusado de las actitudes objeto de este estudio, como predice la teoría del contacto. A falta de un conocimiento más cierto al respecto, se puede decir que las 
prescripciones de la Ley $\mathrm{N}^{\circ} 20845$ son un contexto favorable, pero en ausencia de proyectos pedagógicos que procuren deliberadamente la no discriminación y la tolerancia en las relaciones sociales, ya sea por los esfuerzos instruccionales o regulativos de la escuela, no es evidente que los estudiantes las desarrollen incluso en espacios inclusivos. Esto justifica la realización de más estudios en las escuelas inclusivas actualmente existentes; entre otros, de los factores que explican su presencia en medio de un sistema predominantemente segregado y de las dinámicas de interacción estudiantil en su interior.

\section{Referencias}

Amadeo, J., Torney-Purta, J., Lehmann, R., Husfeldt, V., \& Nikolova, R. (2002): Civic knowledge and engagement: An IEA study of upper secondary students in sixteen countries. Amsterdam: The International Association for the Evaluation of Educational Achievement.

Barlow, F., Paolini, S., Pedersen, A., Hornsey, M., Radke, H., Harwood, J., Rubin, M., \& Sibley, C. (2012). The contact caveat: Negative contact predicts increased prejudice more than positive contact predicts reduced prejudice. Personality and Social Psychology Bulletin, 38, 1629 1643. http://dx.doi.org/10.1177/0146167212457953

Castillo, J. C., Miranda, D., Bonhomme, M., Cox, C., \& Bascopé, M. (2014a). Mitigating the political participation gap from the school: The roles of civic knowledge and classroom climate. Journal of Youth Studies, 18(1), 2-20. http://dx.doi.org/10.1080/13676261.2014.933199

Castillo, J. C., Miranda, D., Bonhomme, M., Cox, C. \& Bascopé, M. (2014b). Social inequality and changes in students' expected political participation in Chile. Education, Citizenship and Social Justice, 9(2), 140-156. http://dx.doi.org/10.1177/1746197914520650

Harris, D. N. (2010). How do school peers influence student educational outcomes? Theory and evidence from economics and other social sciences. Teachers College Record, 112(4), 1163-1197.

Hodson, G. \& Hewstone, M. (2012). Introduction: Advances in intergroup contact. En G. Hodson \& M. Hewstone (Eds.), Advances in intergroup contact (pp. 3-20). New York: Psychology Press.

Iaes, G. y Delich, A. (2009). Sistemas educativos y cohesión social: la reconstrucción de lo común en los Estados nacionales del siglo XXI. En S. Schwartzman y C. Cox, (Eds.), Politicas educativas y cohesión social en América Latina (pp. 179-234). Santiago de Chile: Editorial Uqbar. 
Janmaat, J. G. (2012). The effect of classroom diversity on tolerance and participation in England, Sweden and Germany. Journal of Ethnic and Migration Studies, 38(1), 21-39. http://dx.doi.org/10.1080/136918 $3 \times .2012 .640007$

Janmaat, J. G. (2014). Do ethnically mixed classrooms promote inclusive attitudes towards immigrants everywhere? A study among native adolescents in 14 western countries. European Sociological Review, 30(6), 810-822. http://dx.doi.org/10.1093/esr/jcu075

Janmaat, J. G. (2015). School ethnic diversity and white students' civic attitudes in England. Social Science Research, 49, 97-109. http://dx.doi. org/10.1016/j.ssresearch.2014.07.006

Ministerio de Educación de Chile, Mineduc. (2010). Estudio internacional de educación cívica y formación ciudadana ICCS 2009, primer informe nacional. Santiago de Chile: autor.

Organisation for Economic Co-operation and Development, OECD. (2009). PISA Data analysis manual, SPSS (2ª ed.). Paris: OECD Publishing.

Organisation for Economic Co-operation and Development, OECD. (2010). PISA 2009. Results: Overcoming social background: Equity in learning opportunities and outcomes, Volume II. Paris: OECD Publishing.

Organisation for Economic Co-operation and Development, OECD (2011). Perspectives on global development 2012: Social cohesion in a shifting world. Executive summary. Paris: OECD Publishing. http://dx.doi.org/10.1787/ persp_glob_dev-2012-en.

Organisation for Economic Co-operation and Development, OECD (2013). PISA 2012. Results: Excellence through equity. Giving every student the chance to succeed, Volume II. Paris: OECD Publishing. http://dx.doi. org/10.1787/9789264201132

Paolini, S., Harris, N., \& Griffin, A. (2015). Learning anxiety in interactions with the outgroup: Towards a learning model of anxiety and stress in intergroup contact. Group Processes \& Intergroup Relations, 19(3), 275313. http://dx.doi.org/10.1177/1368430215572265

Paolini, S., Harwood, J., \& Rubin, M. (2010). Negative intergroup contact makes group memberships salient: Explaining why intergroup conflict endures. Personality and Social Psychology Bulletin, 36(12), 1723-1738. http://dx.doi.org/10.1177/0146167210388667

Peña, C. (2007). Por qué no debemos seleccionar. En J. J. Brunner y C. Peña (Eds.), La reforma del sistema escolar: aportes para el debate (pp. 245252). Santiago de Chile: Universidad Diego Portales. 
Pettigrew, T., Tropp, L., Wagner, U., \& Christ, O. (2011). Recent advances in intergroup contact theory. International Journal of Intercultural Relations, 35(3), 271-280. http://dx.doi.org/10.1016/j.ijintrel.2011.03.001

Pettigrew, T. \& Tropp, L. (2006). A meta-analytic test of intergroup contact theory._Journal of Personality and Social Psychology, 90(5), 751783. http://dx.doi.org/10.1037/0022-3514.90.5.751

Putnam, R. (2007). E Pluribus Unum: Diversity and community in the twentyfirst century - The 2006 Johan Skytte Prize Lecture. Scandinavian Political Studies, 30(2), 137-174. http://dx.doi.org/10.1111/j.14679477.2007.00176.x

Schulz, W., Ainley, J., \& Fraillon, J. (Eds.) (2011). ICCS 2009 technical report. Amsterdam: IEA.

Schulz, W., Ainley, J., Fraillon, J., Kerr, D., \& Losito, B. (2010). ICCS 2009 international report: Civic knowledge, attitudes, and engagement among lower-secondary school students in 38 countries. Amsterdam: IEA.

Schulz, W., Ainley, J., Friedman, T., \& Lietz, P. (2011). Informe latinoamericano del ICCS 2009: actitudes y conocimientos cívicos de estudiantes de secundaria en seis países de América Latina. Amsterdam: Asociación Internacional para la Evaluación del Logro Educativo (IEA).

Tajfel, H. (1984). Grupos humanos y categorías sociales. Barcelona: Herder.

Techakesari, P., Barlow, F., Hornsey, M., Sung, B., Thai, M., \& Chak, J. (2015). An investigation of positive and negative contact as predictors of intergroup attitudes in the United States, Hong Kong, and Thailand. Journal of Cross-Cultural Psychology, 46, 454-468. http://dx.doi. org/10.1177/0022022115570313

Torney-Purta, J., Lehmann, R., Oswald, H., \& Schulz, W. (2001): Citizenship and education in twenty-eight countries: Civic knowledge and engagement at age fourteen. Amsterdam: The International Association for the Evaluation of Educational Achievement.

Valenzuela, J. P., Bellei, C. y De Los Ríos, D. (2009). Evolución de la segregación socioeconómica de los estudiantes chilenos y su relación con el financiamiento compartido. En Fondo de Investigación y Desarrollo en Educación (Ed.), Evidencias para políticas públicas en educación (pp. 231-284). Santiago de Chile: Ministerio de Educación.

Recibido: 07/01/2016

Aceptado: 19/05/2016 
96 ACTITUDES DE LOS ESTUDIANTES EN ESCUELAS SEGREGADAS Y EN ESCUELAS INCLUSIVAS, HACIA LA TOLERANCIA SOCIAL Y LA CONVIVENCIA ENTRE PARES - I. Ortiz

\section{ANEXO}

\section{Cuadros de frecuencias relativas de cinco variables dependientes por} tipo de escuela.

Cuadro i. Variable "Aceptación de gente de otra clase social en el vecindario" por tipo de escuela. Frecuencias relativas

\begin{tabular}{l|l|c|c}
\hline Tipo escuela & & Porcentaje & Error estándar \\
\hline \multirow{4}{*}{ Segregada pobre } & Me gustaría & 35,4 & 1,75 \\
\cline { 2 - 4 } & No me importaría & 59,4 & 1,9 \\
\cline { 2 - 4 } & Me disgustaría & 5,2 & 0,57 \\
\hline \multirow{4}{*}{ Inclusiva } & Me gustaría & 37,9 & 1,69 \\
\cline { 2 - 4 } & No me importaría & 57,7 & 1,66 \\
\cline { 2 - 4 } & Me disgustaría & 4,4 & 0,58 \\
\hline \multirow{4}{*}{ Segregada aventajada } & Me gustaría & 33,2 & 1,24 \\
\cline { 2 - 4 } & No me importaría & 61,5 & 1,28 \\
\cline { 2 - 4 } & Me disgustaría & 5,2 & 0,44 \\
\hline
\end{tabular}

Cuadro ii. Variable "Confianza en la gente" por tipo de escuela. Frecuencias relativas

\begin{tabular}{l|c|c|c}
\hline Tipo escuela & & Porcentaje & Error estándar \\
\hline \multirow{2}{*}{ Segregada pobre } & No & 48,5 & 1,69 \\
\cline { 2 - 4 } & Sí & 51,5 & 1,69 \\
\hline \multirow{2}{*}{ Inclusiva } & No & 48,36 & 1,5 \\
\hline \multirow{2}{*}{ Segregada aventajada } & Sí & 51,64 & 1,5 \\
\hline & No & 47,87 & 1,34 \\
\cline { 2 - 4 } & Sí & 52,13 & 1,34 \\
\hline
\end{tabular}

Cuadro iii. Variable "Rechazo a ser incluido en un grupo" por tipo de escuela. Frecuencias relativas

\begin{tabular}{l|c|c|c}
\hline \multirow{2}{*}{ Tipo escuela } & Rechazo & Porcentaje & Error estándar \\
\hline \multirow{2}{*}{ Segregada pobre } & Nunca & 68,46 & 1,66 \\
\cline { 2 - 4 } Inclusiva & Sí & 31,54 & 1,66 \\
\hline \multirow{2}{*}{ Segregada aventajada } & Nunca & 69,76 & 1,51 \\
\cline { 2 - 4 } & Sí & 30,24 & 1,51 \\
\cline { 2 - 4 } & Nunca & 77,08 & 1,29 \\
\cline { 2 - 4 } & Sí & 22,92 & 1,29 \\
\hline
\end{tabular}


Cuadro iv. Variable "Ser objeto de insultos" por tipo de escuela. Frecuencias relativas

\begin{tabular}{l|c|c|c}
\hline Tipo escuela & Insultos & Porcentaje & Error estándar \\
\hline \multirow{2}{*}{ Segregada pobre } & Nunca & 31,79 & 1,69 \\
\cline { 2 - 4 } & Sí & 68,21 & 1,69 \\
\hline \multirow{2}{*}{ Inclusiva } & Nunca & 33,4 & 1,7 \\
\cline { 2 - 4 } & Sí & 66,6 & 1,7 \\
\hline \multirow{2}{*}{ Segregada aventajada } & Nunca & 40,69 & 2,1 \\
\cline { 2 - 4 } & Sí & 59,31 & 2,1 \\
\hline
\end{tabular}

Cuadro v. Variable "Ser objeto de apodos ofensivos" por tipo de escuela. Frecuencias relativas

\begin{tabular}{l|c|c|c}
\hline Tipo escuela & Apodos & Porcentaje & Error estándar \\
\hline \multirow{2}{*}{ Segregada pobre } & Nunca & 38,47 & 1,92 \\
\cline { 2 - 4 } & Sí & 61,53 & 1,92 \\
\hline \multirow{2}{*}{ Inclusiva } & Nunca & 43,49 & 1,73 \\
\cline { 2 - 4 } & Sí & 56,51 & 1,73 \\
\hline \multirow{2}{*}{ Segregada aventajada } & Nunca & 53 & 2,12 \\
\cline { 2 - 4 } & Sí & 47 & 2,12 \\
\hline
\end{tabular}

